\section{UHF RFID Elastic Textile Yarn}

\author{
Sofia Benouakta ${ }^{1}$, Florin $\mathrm{Hutu}^{2}$, Daniele \\ Sette $^{3}$, Yvan Duroc ${ }^{1}$ \\ ${ }^{1}$ Université de Lyon, Université Claude Bernard Lyon \\ 1, CNRS, Ampère, F-69622, Villeurbanne, France. \\ ${ }^{2}$ Univ Lyon, INSA Lyon, Inria, CITI, F-69621, \\ Villeurbanne, France. \\ ${ }^{3}$ Primo1D, 7 parvis Louis Néel, 38040 Grenoble Cedex \\ 9, France. \\ sofia.benouakta@univ-lyonl.fr
}

\begin{abstract}
Over the last decade, the wearable electronics has greatly improved and the wearables market is expected to increase exponentially in the next years. UHF (Ultra High Frequencies) RFID (Radio Frequency IDentification) textile yarns represent one of the most relevant solutions for adding smart functionality into clothes and more generally for any object integrating a textile tag. Among the existing textile yarn solutions, the EThread® technology consists on electronics embedded into textile yarn using a microencapsulation method. This technology is the starting point of the work presented in this paper.

One of the hardest constraints that the RFID yarn should overcome is the robustness against stretching. Thus, the antenna has to be tolerant to the deformation of the associated textile yarn. Unlike the half-wave dipole antenna used in the current E-Thread ${ }^{\circledR}$ yarn, the proposed solution is based on helical dipole antenna topology because its geometry naturally makes feasible an axial stretching.
\end{abstract}

This study details the manufacturing process as well as the design methodology of the antenna taking into account the RFID context as well as the constraints imposed by the manufacturing process. The impact of the antenna's physical parameters is highlighted in order to provide design guidelines. The helical dipole antenna enables until $16 \%$ of axial elongation while the dipole antenna would tear apart.
Keywords: helical antennas, RFID tag, RFID yarn, smart textile.

\section{INTRODUCTION}

E-Textiles or electronics embedded into garments hold a great potential to revolutionize wearable smart objects. The main technological challenges are the extension of advanced garment's functionality, including the introduction of new capabilities such as wireless communication, sensing, actuating, computing and energy harvestings ${ }^{1-2-3-4}$.

Supported by well-established standards and already deployed in wide and various ranges of other applications ${ }^{5-6}$, the UHF RFID systems are of particular interest for contributing to the emergence of smart garments. Textile-based RFID tags have the advantage of the identification functionality and currently offer new functionalities such as sensing for monitoring applications.

In addition to the methods to manufacture 3D and textile RFID tags ${ }^{7-11}$, the concept presented by Vera et al. ${ }^{12}$ is based on the industrial E-Thread® technology ${ }^{13}$. The antenna structure consists in a designed slender and comfortable RFID dipole tag within a wrapped textile wire. This solution re-invented the RFID tag form factor in terms of simplicity of manufacturing process as well as the comfort for users. However, a dipole antenna is fragile when exposed to mechanical constraints, especially to stretching that may lead to a disconnection between the two strands of the dipole and the RFID chip. In order to solve this issue, the presented study proposes to use a helical dipole antenna. Such antenna geometry should enable to keep the expected form factor (i.e., the linear form of a yarn) and be relatively tolerant to a limited stretching while maintaining proper electrical and electromagnetic radiation properties. This type of antenna is not new ${ }^{14}$. Helical dipole antenna recently gained interest: for example, in medical applications as the implantable devices ${ }^{15}$; in RFID field, notably for its advantages in terms of reduced physical size and high inductive impedance. From full-wave 
simulations, the study presented by Liao et al. ${ }^{16}$ shows design steps for a Normal Mode Helical Antenna (NMHA) highlighting the impact of the geometrical parameters of the antenna. An example is detailed for the frequency band 902$928 \mathrm{MHz}$ (i.e., UHF RFID standard in United States) and using a RFID chip with an input impedance equal to $27-j \cdot 201 \Omega$.

In this paper, the goal is to design a UHF RFID textile yarn that is robust in terms of stretching, i.e., a UHF RFID elastic textile yarn. The proposed solution relies on the use of E-Thread $®$ technology and a helical dipole antenna. Compared to the study of Liao ${ }^{16}$, in our work the operating frequency band is narrower 865.5 867.5 MHz (i.e., UHF RFID European frequency band). Moreover, the Monza M4 RFID chip ${ }^{17}$ with input impedance of $15-j \cdot 150 \Omega$ is chosen. This impedance is more representative for current chips and the smaller value of the resistive part is more challenging from the impedance matching perspective.

The paper is organized as follows: Section II presents the manufacturing process of the helical dipole antenna from the E-Thread ${ }^{\circledR}$ yarn. Section III details the design methodology taking into account the specificities to the RFID context and the constraints due to industrial manufacturing. The performance in terms of stretching is notably emphasized including the impact of using a dielectric core material. A comparative study in terms of read range between solutions based on EThread® technology is also presented. In Section IV experimental results are presented and analyzed. Finally, conclusion and future work are given in section $\mathrm{V}$.

\section{MANUFACTURING PROCESS OF THE HELICAL DIPOLE ANTENNA USING E-THREAD TECHNOLOGY}

A helical dipole antenna is composed of two strands of a winded conductive material (Fig. 1) and it is fully characterized by the following parameters:

- $D=$ diameter of the helical dipole antenna

- $a=$ radius of helical conductive material

- $s$ = spacing between turns (center-to-center)

- $N=$ number of turns

- $h=$ axial half-length

Three main steps (Fig. 2) are required for achieving a textile helical dipole antenna based on the manufacturing process of the original EThread® RFID $\operatorname{tag}^{12}$. The modification of the silicon IC (Integrated Circuit) geometry is firstly performed (Fig. 2a). A 3D structure is created at the wafer level by bonding an active wafer and a cover one, which were both specifically processed. After dicing, each E-Thread ${ }^{\circledR}$ IC has grooves on two parallel sides where antenna micro-wires can be inserted. The second step consists in the assembly of the chip onto the antenna wire. This is made on a fully automated special equipment that produces a continuous EThread® filament made of two continuous conductive wires with ICs assembled at fixed interval. Then, a resin is used for mechanical reinforcement (Fig. 2b). The final step consists in the textile packaging that shapes the antenna as a spiral. A wrapping process is used here and the EThread $\AA$ filament is spun around a core yarn. Then, a second textile filament, typically nylon, is spun in the opposite direction to provide textile finishing (Fig. 2c and Fig. 2d). The speed of the core yarn and the rotation speed of the E-Thread ${ }^{\circledR}$ filament define spiral pitch $(s)$, while core yarn dimensions impose the spiral's inner diameter $(D)$. The mechanical behaviour of the final yarn (elongation and spring force) is mainly given by the mechanical properties of the core yarn.

\section{DESIGN OF THE HELICAL DIPOLE ANTENNA}

All the presented simulations are performed using the electromagnetic commercial software CST Microwave Studio® 2018.

In order to obtain a slender yarn that is guaranteeing the manufacturing constraints, the 
diameter of the helix and the radius of the conductive wire to form the helical shape are fixed to $D=1 \mathrm{~mm}$ and $a=0.1 \mathrm{~mm}$. Consequently, the spacing between turns, $s$, the axial half-length, $h$, and the number of turns, $N$, are the geometrical parameters that have to be optimized in order to achieve the antenna's resonance at UHF frequencies and the impedance matching with the RFID chip. Furthermore, the spacing between turns is assumed at least equal to $0.3 \mathrm{~mm}$ notably in order to respect realistic manufacturing process.

\section{A. Design Method}

Although helical dipole antennas are largely used and theoretical developments exist (general theory on the helical dipole antennas ${ }^{18}$ and equations of Kraus ${ }^{14}$ ), there is a lack of reliable design methodology for this kind of antenna even if some tendencies are well-known. The helical dipole antenna's impedance is strongly impacted by the spacing between turns $s$ and its diameter $D$. The increasing of the number of turns decreases the resonance frequency while the input impedance is not strongly impacted, notably at the resonance frequency.

Otherwise, a specific challenge in the RFID field is the matching impedance between the antenna and the chip. The $50 \Omega$ matching is not valid: the antenna input impedance has to be matched with the chip impedance. In order to reduce the manufacturing complexity, for a given RFID chip, the antenna has to be specifically designed. Note that the matching operation is more sensitive for smaller resistive part of the chip from where the chosen Monza M4 chip.

Taking into account these remarks, from preliminary simulations the initially chosen geometrical parameters of the helical dipole antenna are: $N=60$ and $h=18 \mathrm{~mm}$. After this first dimensioning, the design approach is to start by achieving the impedance matching through the spacing $s$. The parameters $N$ and $h$ are varied in order to perform the impedance matching at the resonance frequency. Note that $N$ and $h$ are closely linked when $s$ is fixed $(h=N \cdot s)$, and they are varying reciprocally.

The first step is to fix the matching impedance. For that, $s$ is varied from $0.3 \mathrm{~mm}$ to $0.7 \mathrm{~mm}$ while $N$ is constant. Fig. 3 shows the input impedance of the helical dipole antenna on the Smith chart (with the chip impedance as reference), when the frequency varies between $800 \mathrm{MHz}$ and 1200 MHz. The best reflection coefficient is achieved for $s=0.3 \mathrm{~mm}$ at a frequency of $1105 \mathrm{MHz}$. The second step is to retrieve the resonance frequency in the UHF RFID band. $N$ is increased (while $s$ constant and equal to $0.3 \mathrm{~mm}$ ) until achieving a resonance frequency at $867 \mathrm{MHz}$. Fig. 4 illustrates the shift in terms of resonance frequency when $N$ increases from 60 to 80 (i.e., $h$ increases from $18 \mathrm{~mm}$ to $24 \mathrm{~mm}$ ). Finally, the optimal parameters of the helical dipole antenna are: $s=0.3 \mathrm{~mm}, N=80$, and $h=24 \mathrm{~mm}$, such as the antenna input impedance is equal to $15+$ $j \cdot 150 \Omega$ at $867 \mathrm{MHz}$.

\section{B. Simulation Results}

Fig. 5 shows the reflection coefficient of the optimized helical dipole antenna. The impedance matching with the RFID chip is achieved at the resonance frequency of $867 \mathrm{MHz}$. The bandwidth (defined at $-10 \mathrm{~dB}$ ) is equal to $6.94 \mathrm{MHz}$, from 863.54 MHz to $870.47 \mathrm{MHz}$.

Fig. 6a and Fig. 6b shows the H-plane and Eplane radiation patterns of the optimized helical dipole antenna. As expected, the radiation pattern is omnidirectional and similar to that of a dipole antenna. For the normal mode, the realized gain is equal to $-1 \mathrm{dBi}$. The axial ratio defined as $A R=E_{\theta}$ $/ E_{\varphi}=30 \mathrm{~dB}$, shows an elliptical polarization.

\section{Robustness in Terms of Axial Stretching}

In order to evaluate the robustness of the helical dipole antenna considering an axial stretching, the half-length $h$ is extended symmetrically for the two sides, from the initial value $h=h_{0}=24 \mathrm{~mm}$ up to $30 \mathrm{~mm}$. In this case, the number of turns is kept constant. Table 1 summarizes the considered lengths and highlights the corresponding relative stretching defined as: 


$$
E[\%]=\frac{h-h_{0}}{h_{0}} \times 100 \%
$$

Fig. 7 shows the impact on the reflection coefficient when $h$ increases. The resonance frequency is shifted toward lower frequencies. The absolute value of the reflection coefficient decreases with an enlargement of the bandwidth. For an axial stretching until $16 \%$, the frequency bandwidth is large enough to ensure the RFID communication link in UHF European band.

\section{Impact of the Dielectric Permittivity of the Antenna Core}

In a more realistic context, the helical dipole antenna is manufactured around a material having a dielectric permittivity that could impact the antenna's characteristics. Fig. 8 shows the variation of the reflection coefficient when the relative permittivity of the antenna core varies from 1 to 3 . The resonance frequency decreases when the dielectric permittivity increases, and so, according to the application, with the same design method this parameter should be introduced during the design process. The dielectric permittivity of the antenna core could offer an additional degree of freedom for the design. This also enables to add a sensing capability to the tag if the permittivity of the antenna core is sensitive to the temperature or the humidity.

\section{E. Comparison in terms of Read Range with other RFID Yarns Based on E-Thread ${ }^{\circledR}$ Technology}

In RFID practice, one of the most important performance criteria is the read range, $r$. From the Friis transmission equation, $r$ can be calculated as:

$r=\frac{\lambda}{4 \pi} \sqrt{\frac{P_{t} G_{t} G_{r} \tau}{P_{t h}}}$

where $\lambda$ is the wavelength, $P_{t}$ is the power transmitted by the reader, $G_{t}$ is the transmitting antenna gain, $G_{r}$ is tag antenna gain, $P_{t h}$ is the tag activation threshold, and $\tau$ is the power transmission coefficient given by: $\tau=\frac{4 R_{\text {chip }} R_{\text {ant }}}{\left|Z_{\text {chip }}+Z_{\text {ant }}\right|^{2}}$

where $Z_{\text {chip }}=R_{c}+j X_{c}$ is the chip impedance $Z_{a}=R_{a}+j X_{a}$ is the antenna input impedance.

Assuming that $G_{t}, P_{t}$ and $P_{t h}$ are constant, it is worth noting that the read range only depends on $G_{r}$ and $\tau$ ( $\tau$ translating the quality of the impedance matching). $G_{r}$ and $\tau$ are two keyparameters for the tag design and they contribute to the read range in an equivalent way in (2).

From this point of view, the gain of - $1 \mathrm{dBi}$ of the helical dipole antenna is a weakness compared to the $2 d B i$ gain of a dipole. In order to compare the performance in terms of read range, two other E-Thread® yarns are considered. The first one is the $172 \mathrm{~mm}$ standard center-fed half-wave dipole with a gain of $2 \mathrm{dBi}$ and an input impedance of $73+j \cdot 43 \Omega$ at the resonance frequency; the second one is the improved version ${ }^{12}$ of the latter including a gamma matching technique to the detriment of $0.4 \mathrm{dBi}$ for the gain.

Considering that the RFID reader has a $E I R P=3.28 \mathrm{~W}$ (EIRP stands for Equivalent Isotropic Radiated Power; $E I R P=P_{t} G_{t}$ ) and $P_{t h}=-17 \mathrm{dBm}$, the transmission coefficient $\tau$ and the read range $r$ were calculated. The results are shown in Fig. 9, Fig. 10 and Fig. 11. The maximum read ranges for the three tags are summarized in Table 2. In terms of read range, the good impedance matching of the helical dipole antenna enables to compensate its low gain compared to the dipole solutions. The relative narrow frequency bandwidth also explains the observed result and can be seen as an improvement point for the future.

\section{EXPERIMENTAL RESULTS}

A first prototype of UHF RFID yarn based on helical dipole antenna was manufactured according to the assembling process described in section II. The prototype shown in Fig. 12 is cascaded with other RFID tags and the overall is wound forming a spool. 
Using a commercial RFID test bench ${ }^{19}$, the estimated read range function of the transmitted frequency is extracted (Fig. 13). The maximum read range of $7.1 \mathrm{~m}$ is obtained at $1040 \mathrm{MHz}$ while the read range in the UHF RFID frequency band is $1 \mathrm{~m}$. Even if the prototype is operational, its performance is strongly degraded. The observed frequency shift is due to the manufacturing process, during the final step where a textile wrapping is used in order to obtain the spiral shape of the antenna. Indeed, considering our manufacturing process, it was not possible to obtain the spacing between the turns $(s)$ smaller than $0.7 \mathrm{~mm}$ in order to have a high accuracy. The consequence was the decrease of the number of turns $(N)$ and so the decrease of the effective length of the helical dipole antenna. Finally in practice, the resulting geometrical parameters of the antenna become: $D=1.8 \mathrm{~mm}, h=24 \mathrm{~mm}$, $N=34.5, s=0.7 \mathrm{~mm}$. In addition, the dielectric permittivity of the yarn core and the textile packaging are $\varepsilon_{r}=3.6$ and $\varepsilon_{r}=2.8$ respectively. In order to validate the design methodology, the fabricated prototype was simulated taking into account its real characteristics (Fig. 14). Fig. 15 shows the reflection coefficient that reveals a resonant frequency at $1040 \mathrm{MHz}$.

Finally, two improvement solutions are possible in order to reduce the resonance frequency to the UHF RFID band: 1) assuming the same manufacturing constraint $(s \geq 0.7 \mathrm{~mm})$, it would be simply required to increase the antenna length (i.e., $N$ ); post-simulation shows that $N=42.4$ will lead to the expected resonance frequency; 2) or obviously, having a manufacturing process that enables to respect the initial value of the spacing between the turns (i.e., $s=0.3 \mathrm{~mm}$ ), value assumed realistic even in practice.

\section{CONCLUSION}

This paper presents the design of a UHF RFID elastic textile yarn. The proposed solution is an alternative to the RFID textile yarn based on EThread ${ }^{\circledR}$ technology. The objective is to offer robustness in terms of axial stretching maintaining the form factor of a yarn by using a helical dipole antenna.

Simulation results have shown good performance in terms of read range $(\sim 10 \mathrm{~m})$ and a possible elongation of $16 \%$. The length of the antenna is also reduced: $48 \mathrm{~mm}$ versus $172 \mathrm{~mm}$. Due to manufacturing constraints, the resonant frequency of the fabricated prototype is shifted but the performance is validated by reverseengineering, i.e., simulations taking into account the real characteristics of the prototype.

The proposed solution based on the combination of E-Thread ${ }^{\circledR}$ technology and welldesigned helical dipole antenna: i) enables to maintain the form factor of a yarn; ii) presents good performance in terms of read range for UHF RFID applications; and iii) seems to be a very promising solution when axial stretching constraints appear.

\section{Acknowledgment}

The presented study, part of the RATFING project, has been supported by « La Région Auvergne Rhône-Alpes » Lyon, France and has been realized in collaboration with Primo1D company.

\section{References}

1. Corchia L, Monti G, Tarricone L. Wearable antennas: nontextile versus fully textile solutions. IEEE Antennas and Propagation Magazine, April 2019; 61:71-83.

2. Lemey S, Agneessens S, Rogier S. Wearable smart objects. IEEE Microwave Magazine, August 2018; 27: 83-100.

3. Benouakta S, Hutu F, Duroc Y. New approaches for augmented UHF RFID textile yarn. IEEE International Conference on RFID Technology and Applications (RFID-TA), September 2019; 136140.

4. Abbas SM, Desai S, Esselle KP, Volakis JL, Hashmi RM. Design and Characterization of a Flexible Wideband Antenna Using Polydimethylsiloxane Composite Substrate. International Journal of Antennas and Propagation, February 2018; 2018: 1-6.

5. Yan L, Zhang Y, Yang L, Ning H. The internet of things: from RFID to the next-generation pervasive 
networked systems (Wireless Networks and Mobile Communications), CRC Press; 2008. 336 p.

6. Duroc Y, Tedjini S. RFID: a key technology for humanity. Comptes Rendus Physique, February 2018; 19: 64-71.

7. Koski K, Sydänheimo L, Rahmat-Samii Y, Ukkonen L. Fundamental characteristics of electrotextiles in wearable UHF RFID patch antennas for body-centric sensing systems. IEEE Transactions on Antennas and Propagation, December 2014; 62: 6454-6462.

8. Patron D, Mongan W, Kurtzweg TP, Fontecchio A, Dion G, Anday EK, Dandekar KR. On the use of knitted antennas and inductively coupled RFID tags for wearable applications. IEEE Transactions on Biomedical Circuits and Systems, December 2016; 10: 1047-1057.

9. Liu Q, Le T, He S, Tentzeris MM. Button-shaped radio-frequency identification tag combining threedimensional and inkjet printing technologies. IET Microwave Antennas Propagation, 2016; 10: 737 741.

10. Chen X, Ma S, Ukkonen L, Björninen T, Virkki J. Antennas and antenna-electronics interfaces made of conductive yarn and paint for cost-effective wearable RFIDs and sensors. Proceedings of the IEEE MTT-S International Microwave Symposium, June 2017; 827-830.

11. Shao S, Kiourti A, Burkholder RJ, Volakis JL. Broadband Textile-Based Passive UHF RFID Tag Antenna for Elastic Material. IEEE Antennas and Wireless Propagation Letters, 2015; 14: $1385-$ 1388.

12. Vera G. Slenderly and conformable passive UHF RFID yarn. Proceedings of the IEEE International Conference on RFID, May 2017; 130-136.

13. Brun J. Method for assembling a microelectronic chip element on a wire element, and installation enabling assembly to be performed. US Patent App, January 2015 14/375, 859.

14. Kraus JD. The helical antenna. Proceedings of the I.R.E, March 1949; 37: 263-272.

15. Mizuno H, Takahashi M, Saito K, Haga N, Ito K. Design of a helical folded dipole antenna for biomedical implants. Proceedings of the $5^{\text {th }}$ European Conference on Antennas and Propagation, April 2011; 3484-3487.

16. Liao Y, Zhang Y, Cai K, Liang Z. Impedance matching design of small normal mode helical antennas for RFID tags. Proceedings of the International Symposium on Antennas \& Propagation, 2013; 1150-1153.

17. Impinj. Impinj UHF RFID product downloads monza tag chips Available: http://www.impinj.com/products/tag-chips/
18. Balanis CA, Antenna Theory: Analysis and Design, Wiley; 2005. $1136 \mathrm{p}$

19. Voyantic. Tagformance pro. [Online]. Available: https://voyantic.com/products/tagformance-pro

\section{TABLES}

\begin{tabular}{|c|c|c|c|c|}
\hline$h[\mathrm{~mm}]$ & 24 & 26 & 28 & 30 \\
\hline$E[\%]$ & 0 & 8.3 & 16 & 25 \\
\hline
\end{tabular}

Table 1. Relative elongation for each considered length.

\begin{tabular}{|c|c|c|c|}
\hline Tag & $\begin{array}{c}\text { Simple } \\
\text { dipole }\end{array}$ & $\begin{array}{c}\text { Matched } \\
\text { dipole }\end{array}$ & $\begin{array}{c}\text { Helical } \\
\text { antenna }\end{array}$ \\
\hline $\begin{array}{c}\text { Maximum read } \\
\text { range (meters) }\end{array}$ & 8.2 & 11.2 & 9.9 \\
\hline
\end{tabular}

Table 2. Maximum read range for the three configurations.

\section{FIGURE LEGENDS}

Fig. 1. Helical dipole antenna: (a) illustration of the design geometrical parameters; (b) full view of the antenna in CST Microwave Studio®; (c) zoomed view at the feeding point.

Fig. 2. Manufacturing process of the helical dipole antenna: (a) E-Thread® IC on Impinj Monza; (b) E-Thread ${ }^{\circ}$ IC assembled on stranded conductive antenna wires; (c) modified wrapping process for spiral E-Thread $®$ yarn manufacturing; (d) X-ray image of E-Thread ${ }^{\circledR}$ filament after spiral process.

Fig. 3. Simulated input impedance of the helical dipole antenna when the frequency is varying between $800 \mathrm{MHz}$ and $1200 \mathrm{MHz}$ for a spacing between turns varying from $0.3 \mathrm{~mm}$ to 0.7 $\mathrm{mm}$ (note that the Smith chart is normalized to the chip's impedance).

Fig. 4. Simulated reflection coefficient $S_{11}$ of the helical dipole antenna for different lengths values from $18 \mathrm{~mm}$ to $24 \mathrm{~mm}$ for achieving resonance frequency in the RFID UHF band.

Fig. 5. Simulated reflection coefficient $S_{11}$ of the helical dipole antenna after the design optimization with: $h=24 \mathrm{~mm}, s=0.3 \mathrm{~mm}, D=1$ $m m, a=0.1 \mathrm{~mm}$. 
Fig. 6. Simulated radiation pattern of the helical dipole antenna after the design optimization. The antenna is placed along $z$ axis: (a) H-plane for $\theta=90^{\circ}$; (b) E-plane for $\varphi=90^{\circ}$.

Fig. 7. Simulated reflection coefficient $S_{11}$ for several values of the axial half-length keeping the spacing between turns fixed, simulating the effect of a stretching.

Fig. 8. Simulated reflection coefficient $S_{11}$ for several relative permittivity $\varepsilon_{r}$ values of the dielectric material constituting the antenna core.

Fig. 9. Simulated read range versus transmission coefficient for: simple dipole, gamma matched dipole and helical dipole antenna.

Fig. 10. Simulated transmission coefficient $\tau$ of the three UHF RFID yarn solutions: simple dipole, gamma matched dipole and helical dipole antenna.

Fig. 11. Simulated read range calculated for the three UHF RFID yarn solutions: simple dipole, gamma matched dipole and helical dipole antenna.

Fig. 12. Manufactured prototype of the helical dipole antenna for UHF RFID yarn cascaded with other RFID tags, the overall is wound forming a spool. Each RFID yarn is separated by cutting.

Fig. 13. Measured read range of the helical dipole antenna.

Fig. 14. Geometrical parameters of the simulated manufactured helical dipole antenna: (a) full view of the structure in CST Microwave Studio®; (b) sectional view of the structure; (c) front view of the structure.

Fig. 15. Simulated reflection coefficient $S_{11}$ of the manufactured helical dipole antenna. 


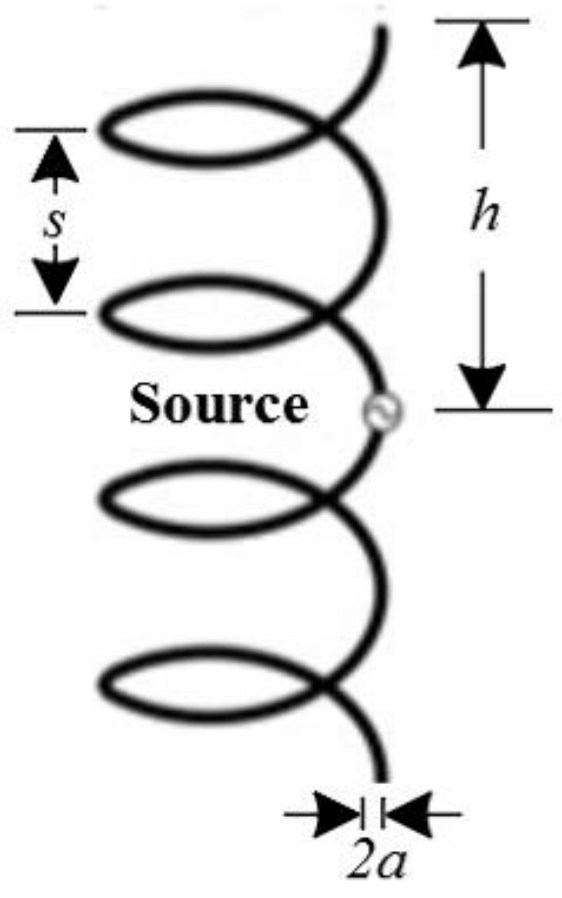

(a)

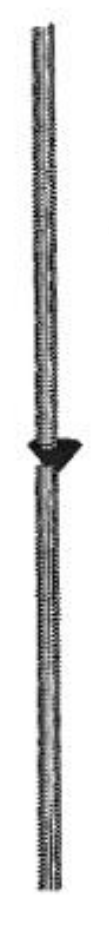

(b)
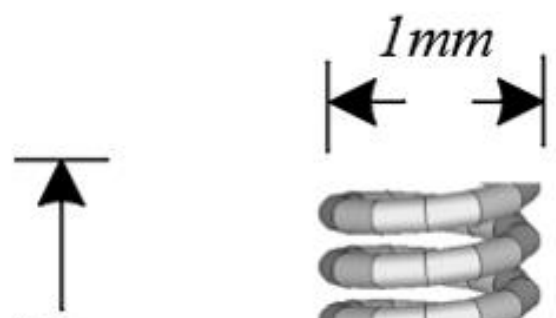

$24 \mathrm{~mm}$
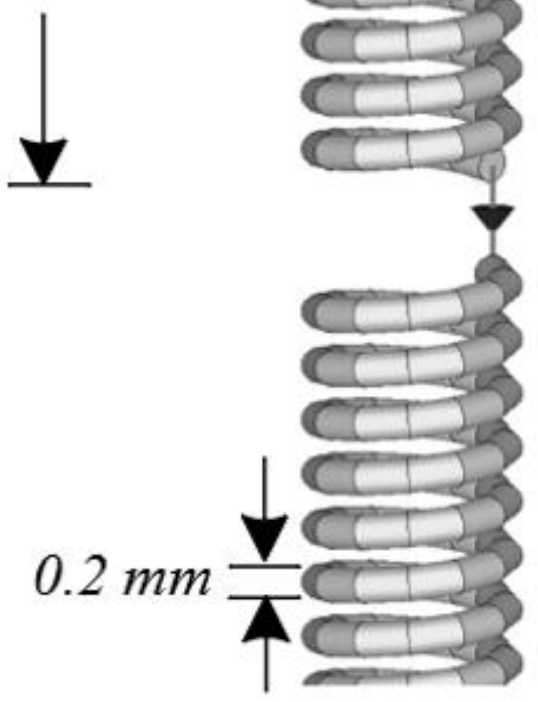

(c)

Fig. 1. Helical dipole antenna: (a) illustration of the design geometrical parameters; (b) full view of the antenna in CST Microwave Studio®; (c) zoomed view at the feeding point. 


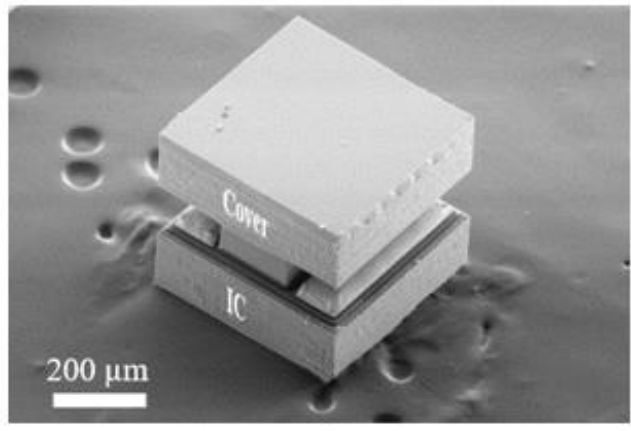

(a)

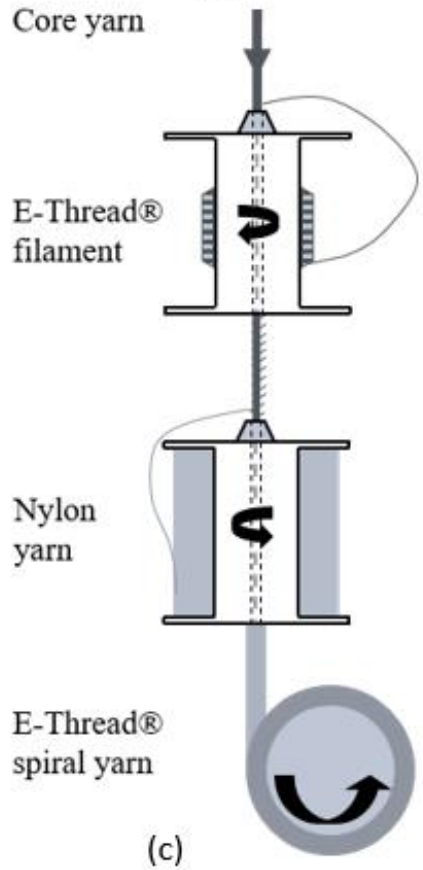

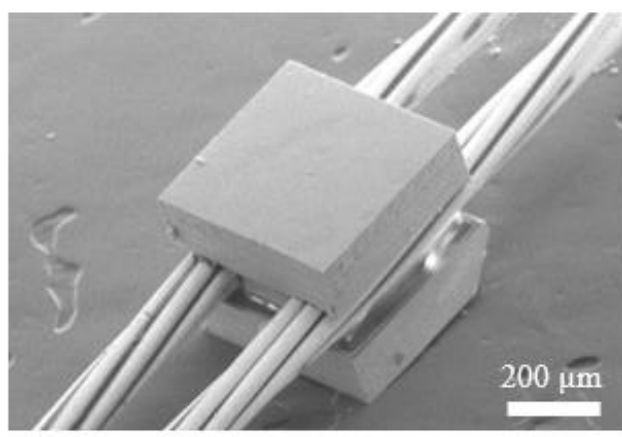

(b)

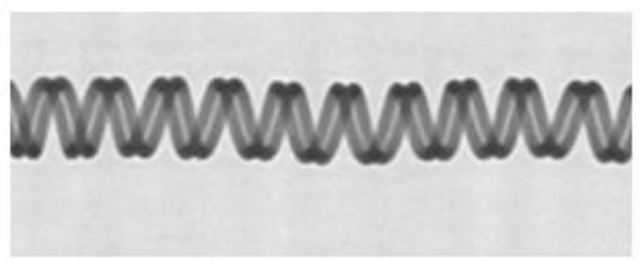

(d)

Fig. 2. Manufacturing process of the helical dipole antenna: (a) E-Thread® IC on Impinj Monza; (b) EThread® IC assembled on stranded conductive antenna wires; (c) modified wrapping process for spiral E-Thread ${ }^{\circledR}$ yarn manufacturing; (d) X-ray image of E-Thread ${ }^{\circledR}$ filament after spiral process. 


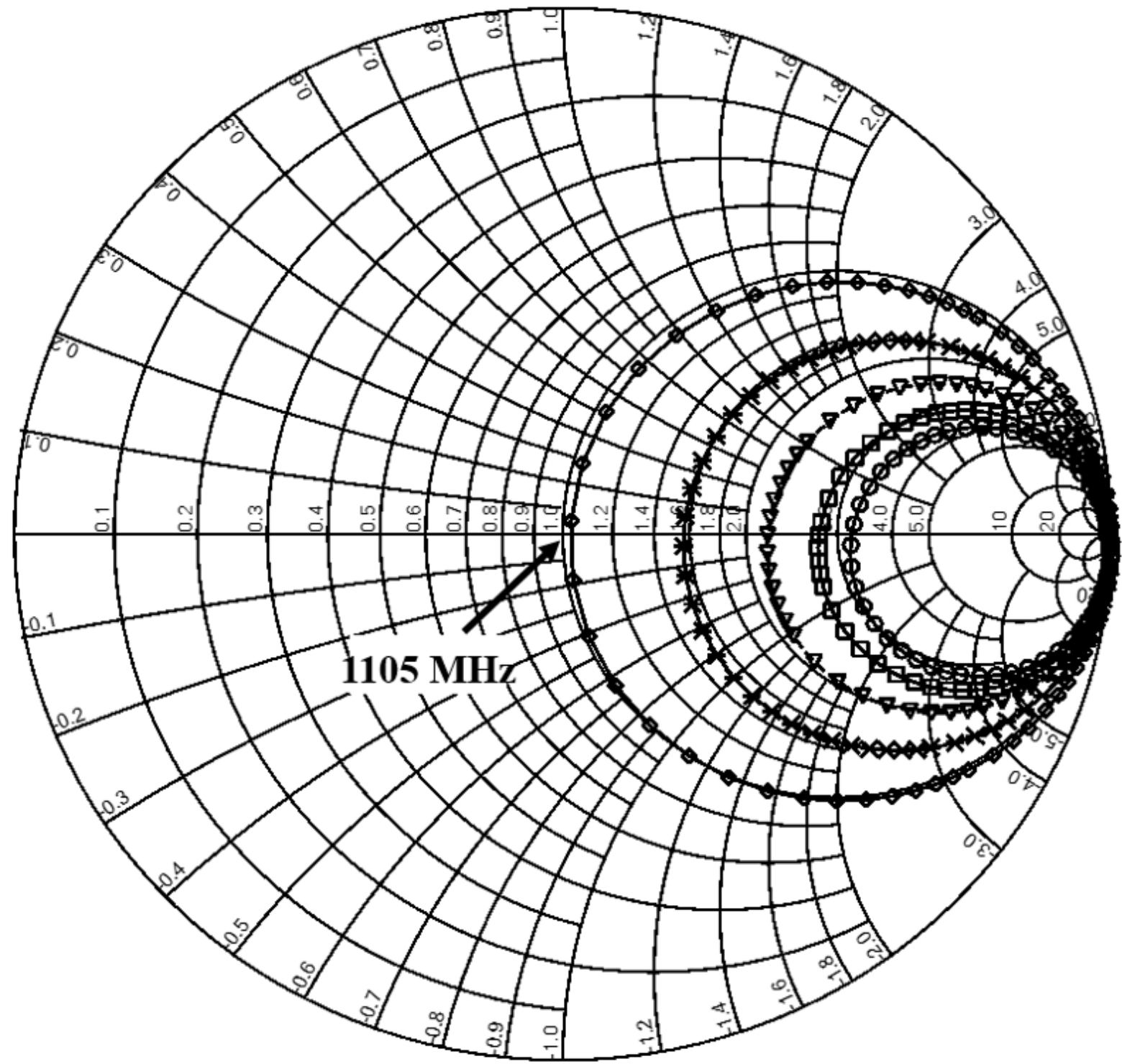

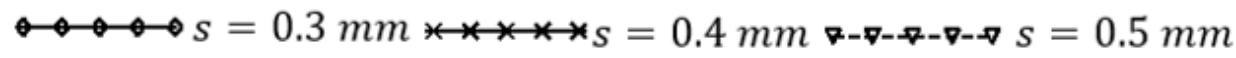

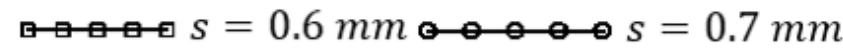

Fig. 3. Simulated input impedance of the helical dipole antenna when the frequency is varying between $800 \mathrm{MHz}$ and $1200 \mathrm{MHz}$ for a spacing between turns varying from $0.3 \mathrm{~mm}$ to $0.7 \mathrm{~mm}$ (note that the Smith chart is normalized to the chip's impedance). 


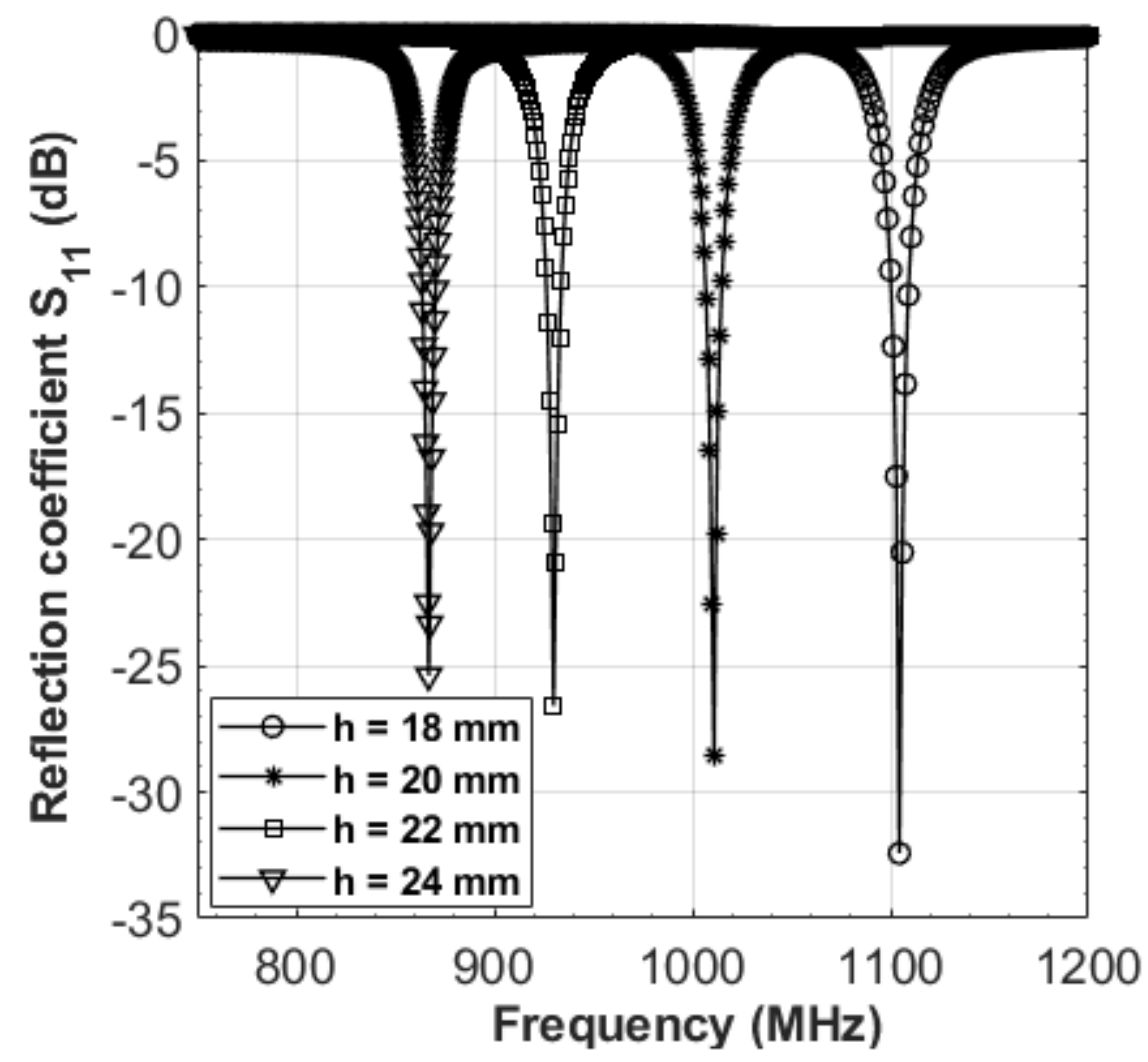

Fig. 4. Simulated reflection coefficient $S_{11}$ of the helical dipole antenna for different lengths values from $18 \mathrm{~mm}$ to $24 \mathrm{~mm}$ for achieving resonance frequency in the RFID UHF band.

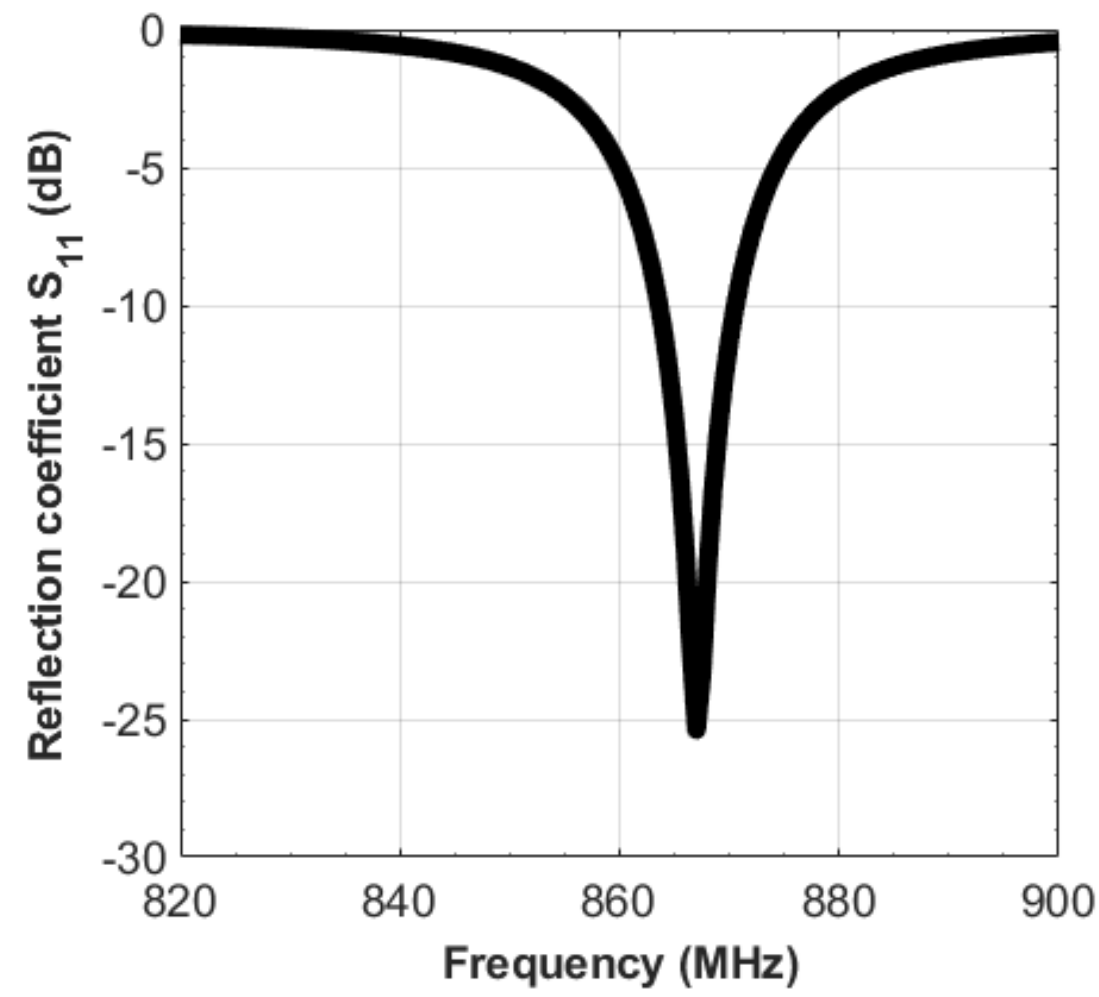

Fig. 5. Simulated reflection coefficient $S_{11}$ of the helical dipole antenna after the design optimization with: $h=24 \mathrm{~mm}, s=0.3 \mathrm{~mm}, D=1 \mathrm{~mm}, a=0.1 \mathrm{~mm}$. 


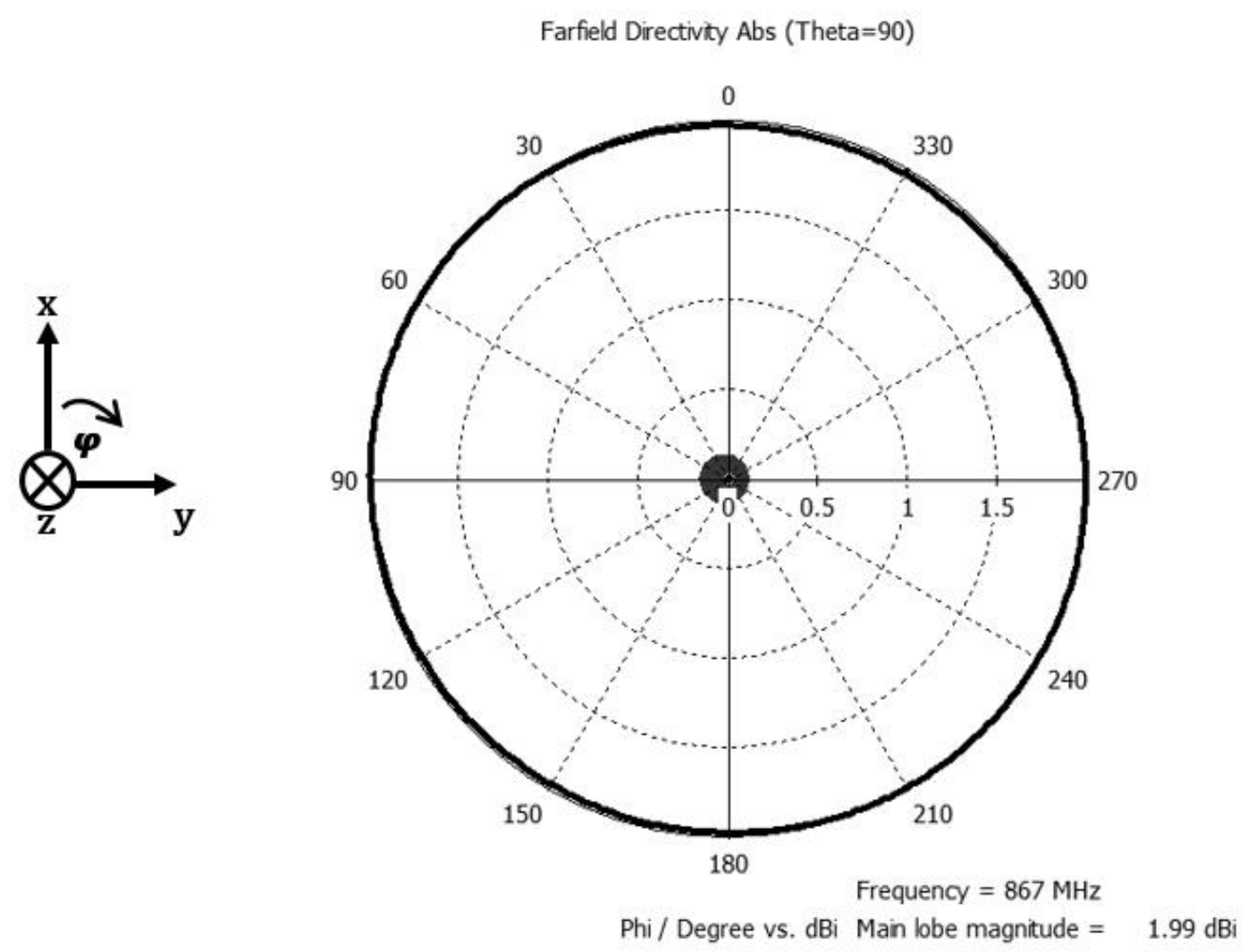

(a)

Farfield Directivity Abs (Phi=90)

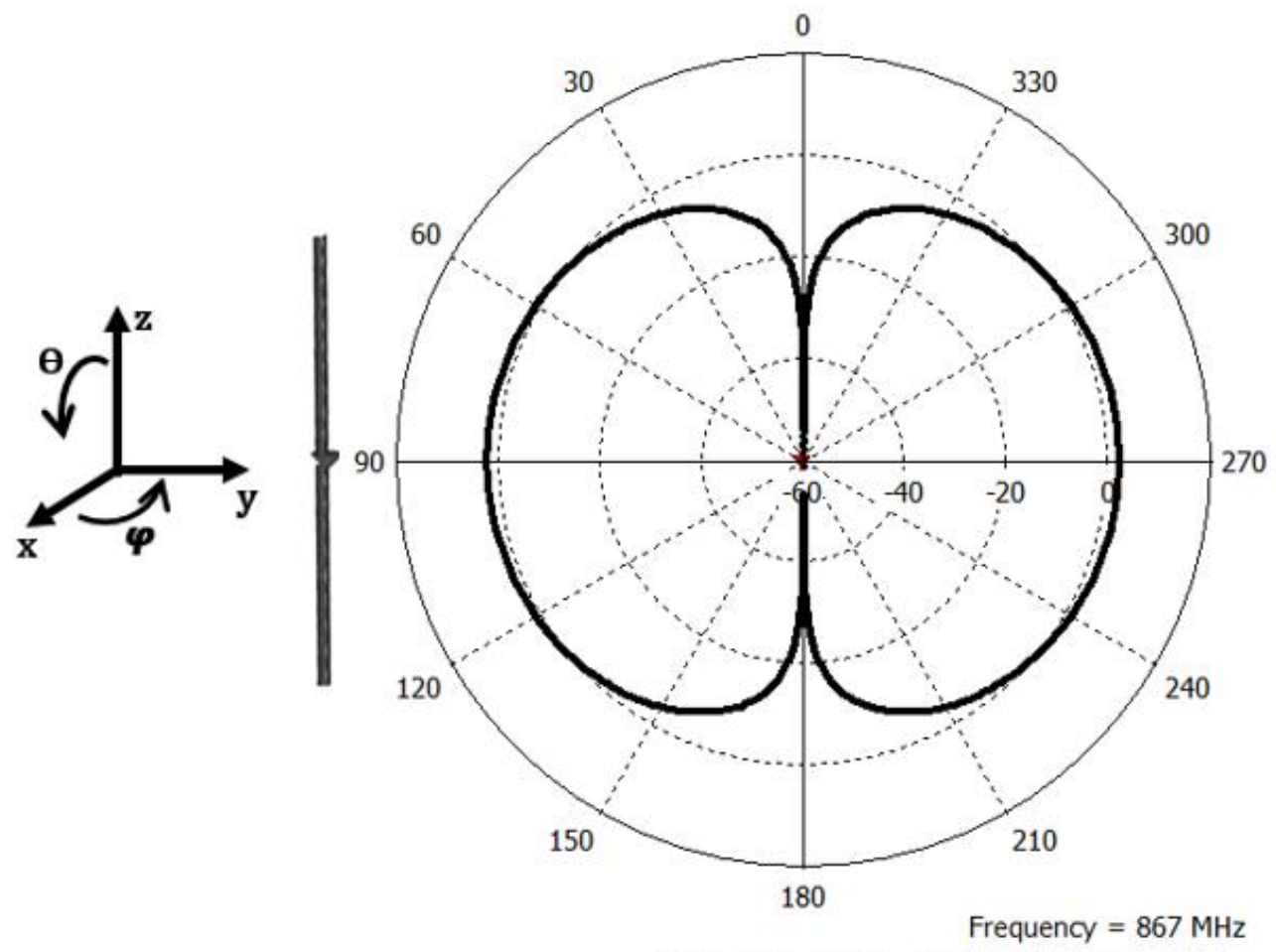

Theta $/$ Degree vs. dBi Main lobe magnitude $=1.99 \mathrm{dBi}$

(b)

Fig. 6. Simulated radiation pattern of the helical dipole antenna after the design optimization. The antenna is placed along $z$ axis: (a) H-plane for $\theta=90^{\circ}$; (b) E-plane for $\varphi=90^{\circ}$. 


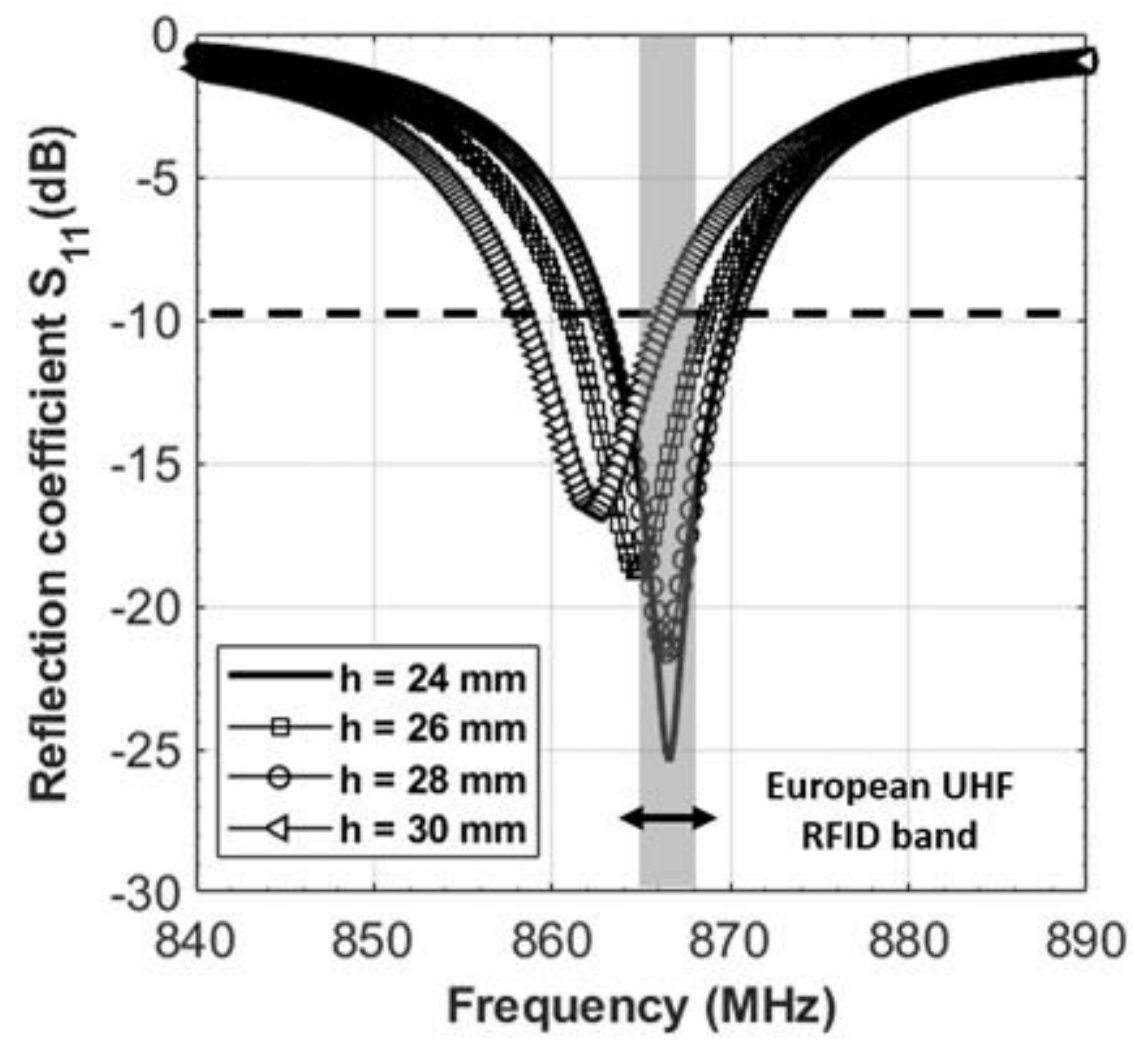

Fig. 7. Simulated reflection coefficient $S_{11}$ for several values of the axial half-length keeping the spacing between turns fixed, simulating the effect of a stretching.

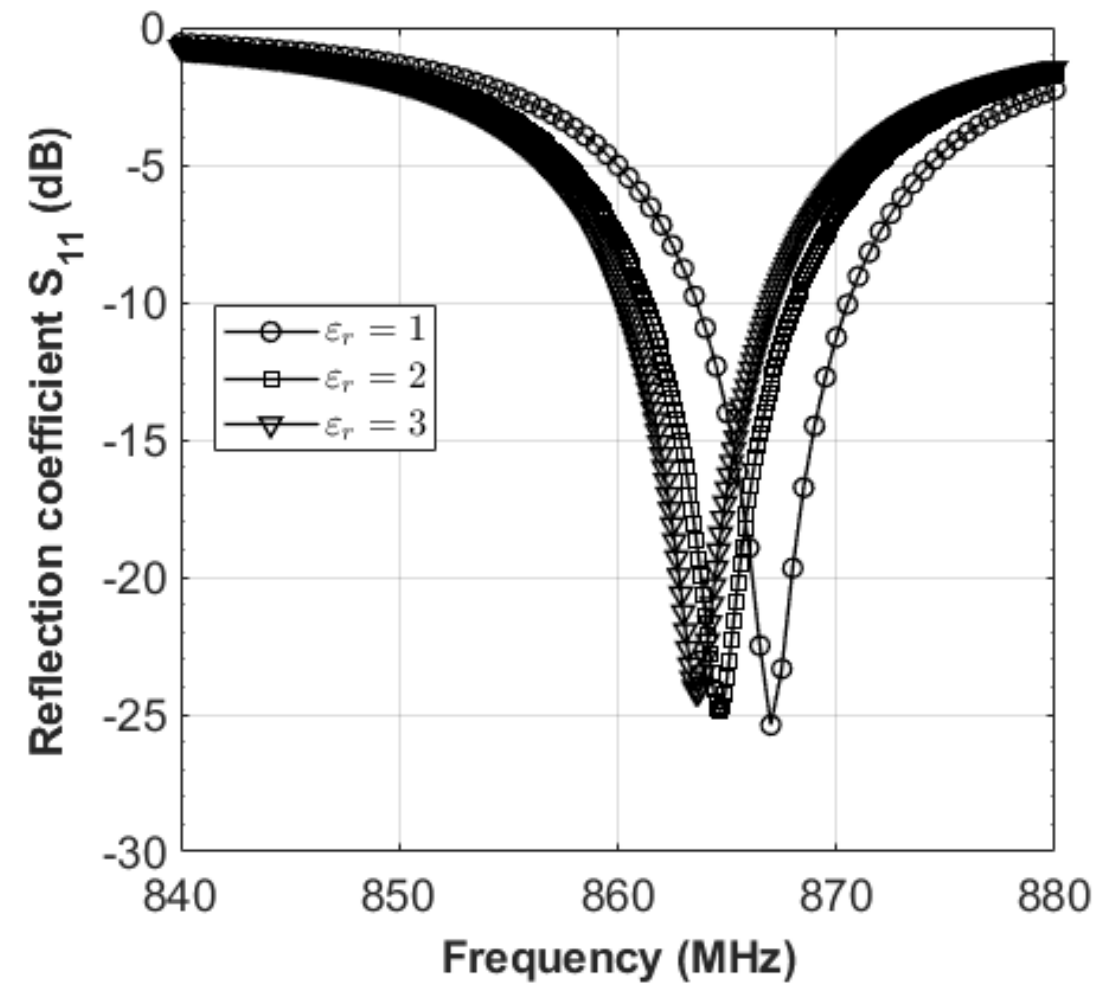

Fig. 8. Simulated reflection coefficient $S_{11}$ for several relative permittivity $\varepsilon_{r}$ values of the dielectric material constituting the antenna core. 


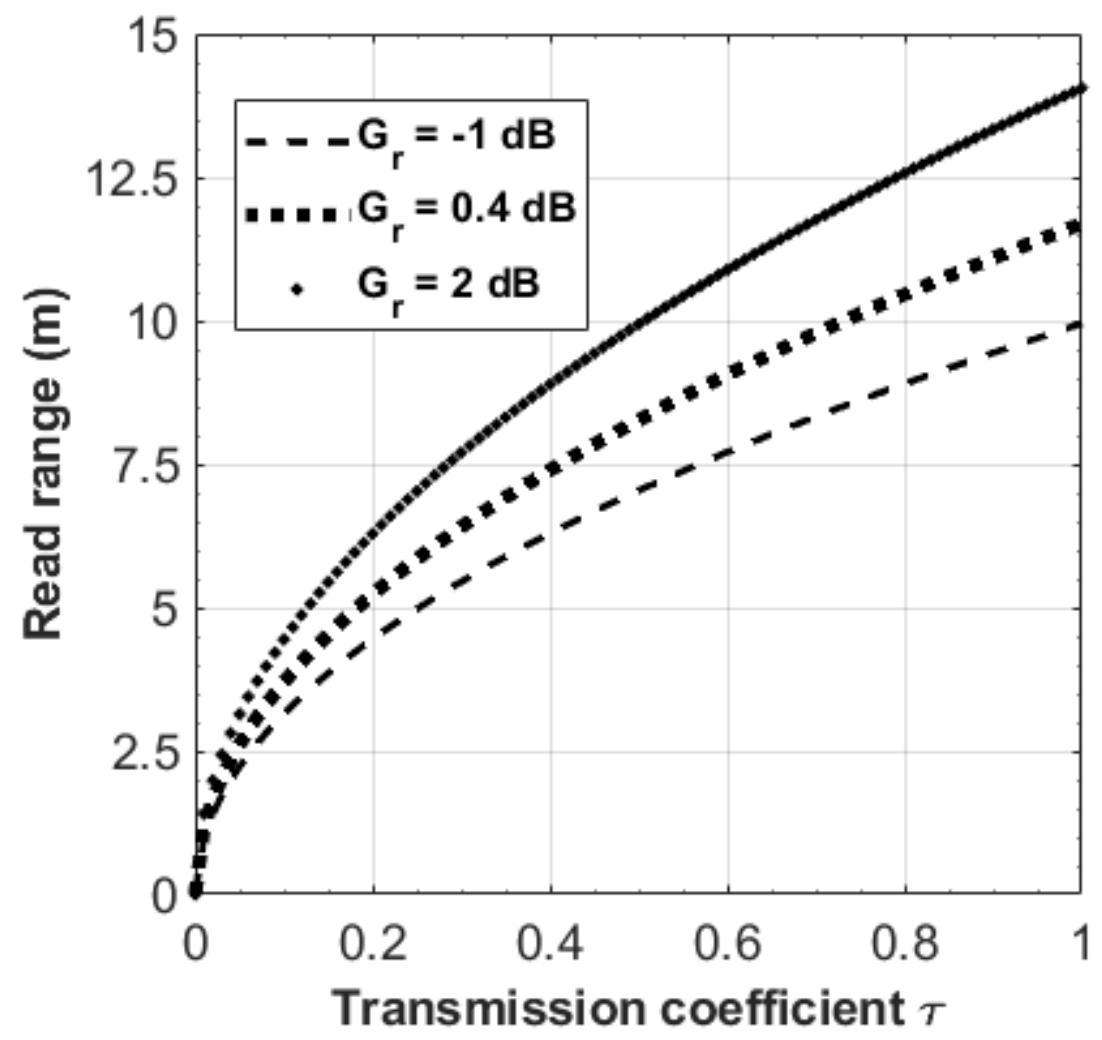

Fig. 9. Simulated read range versus transmission coefficient for: simple dipole, gamma matched dipole and helical dipole antenna.

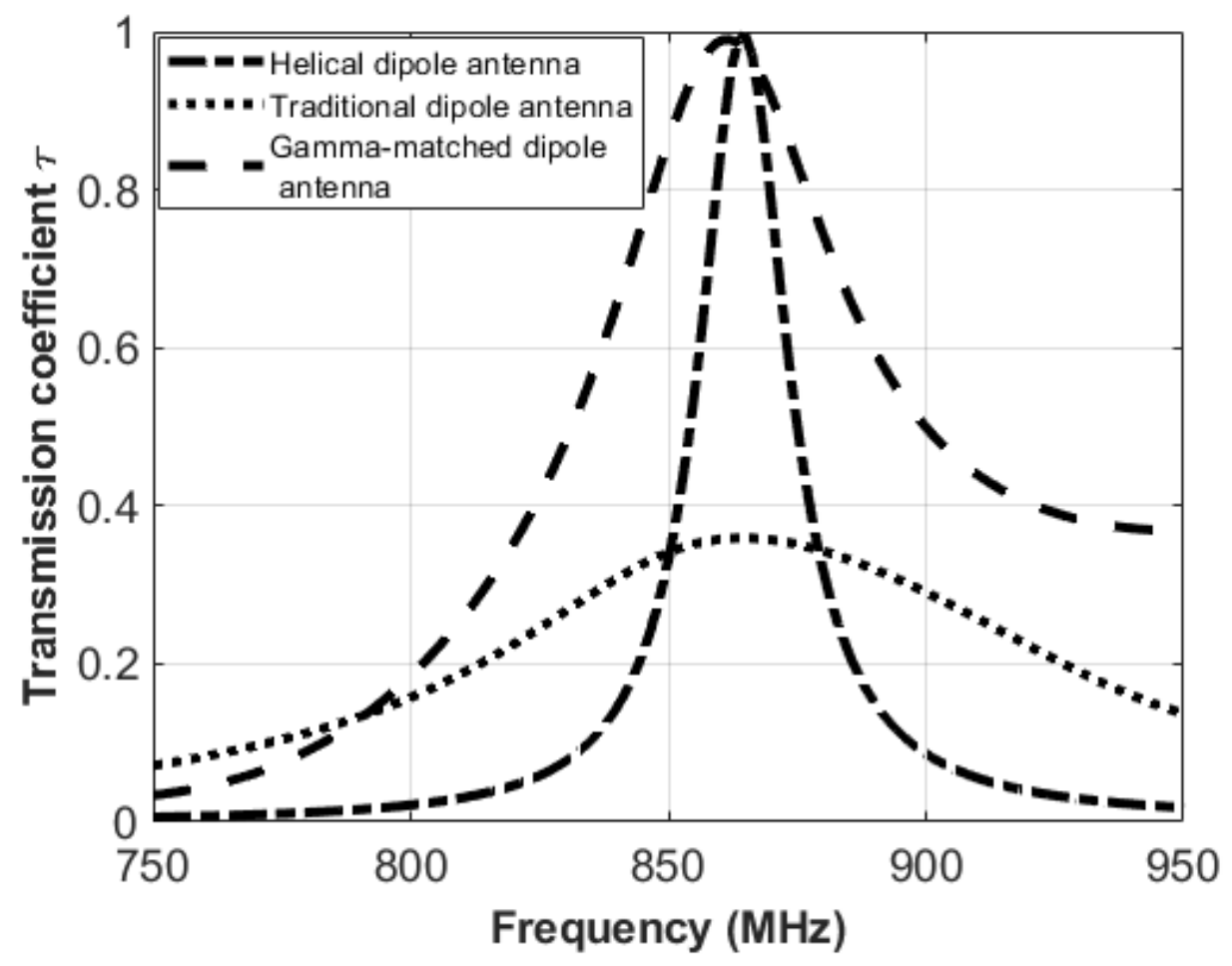

Fig. 10. Simulated transmission coefficient $\tau$ of the three UHF RFID yarn solutions: simple dipole, gamma matched dipole and helical dipole antenna. 


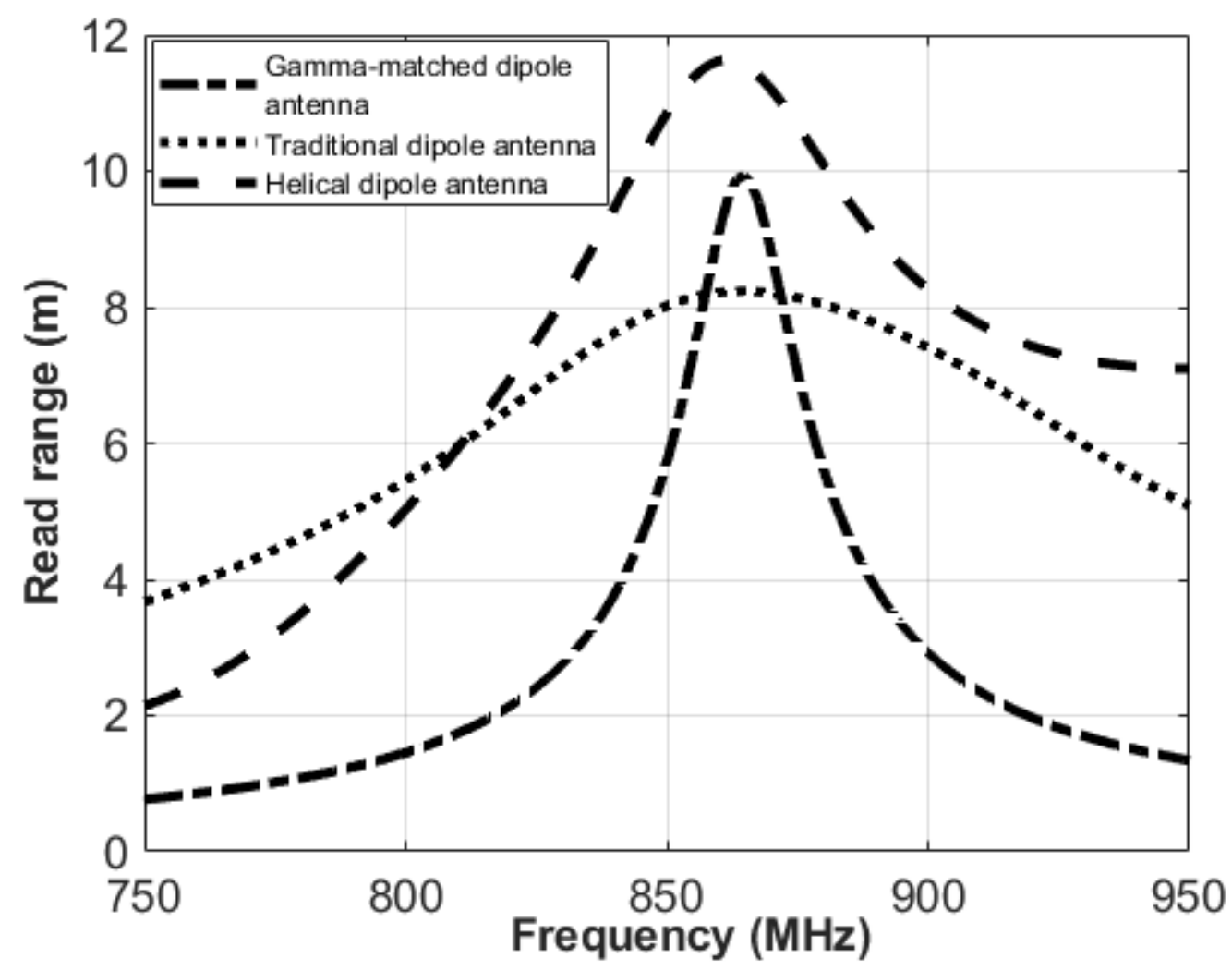

Fig. 11. Simulated read range calculated for the three UHF RFID yarn solutions: simple dipole, gamma matched dipole and helical dipole antenna.

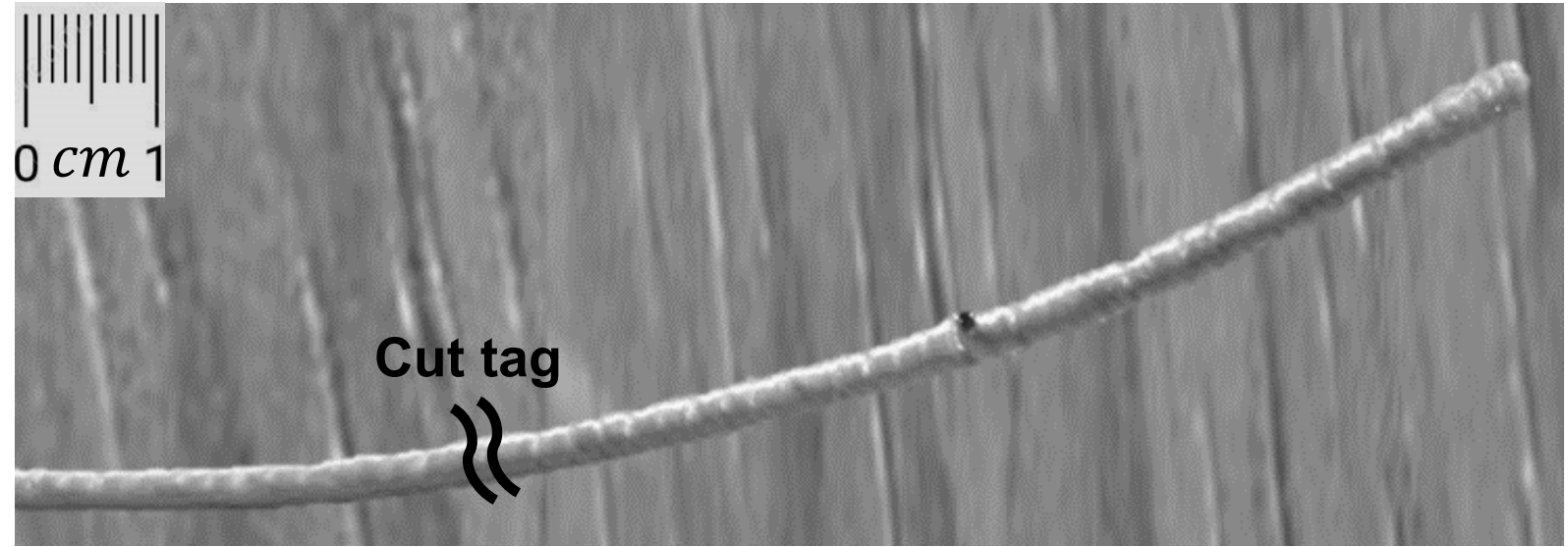

Fig. 12. Manufactured prototype of the helical dipole antenna for UHF RFID yarn cascaded with other RFID tags, the overall is wound forming a spool. Each RFID yarn is separated by cutting. 


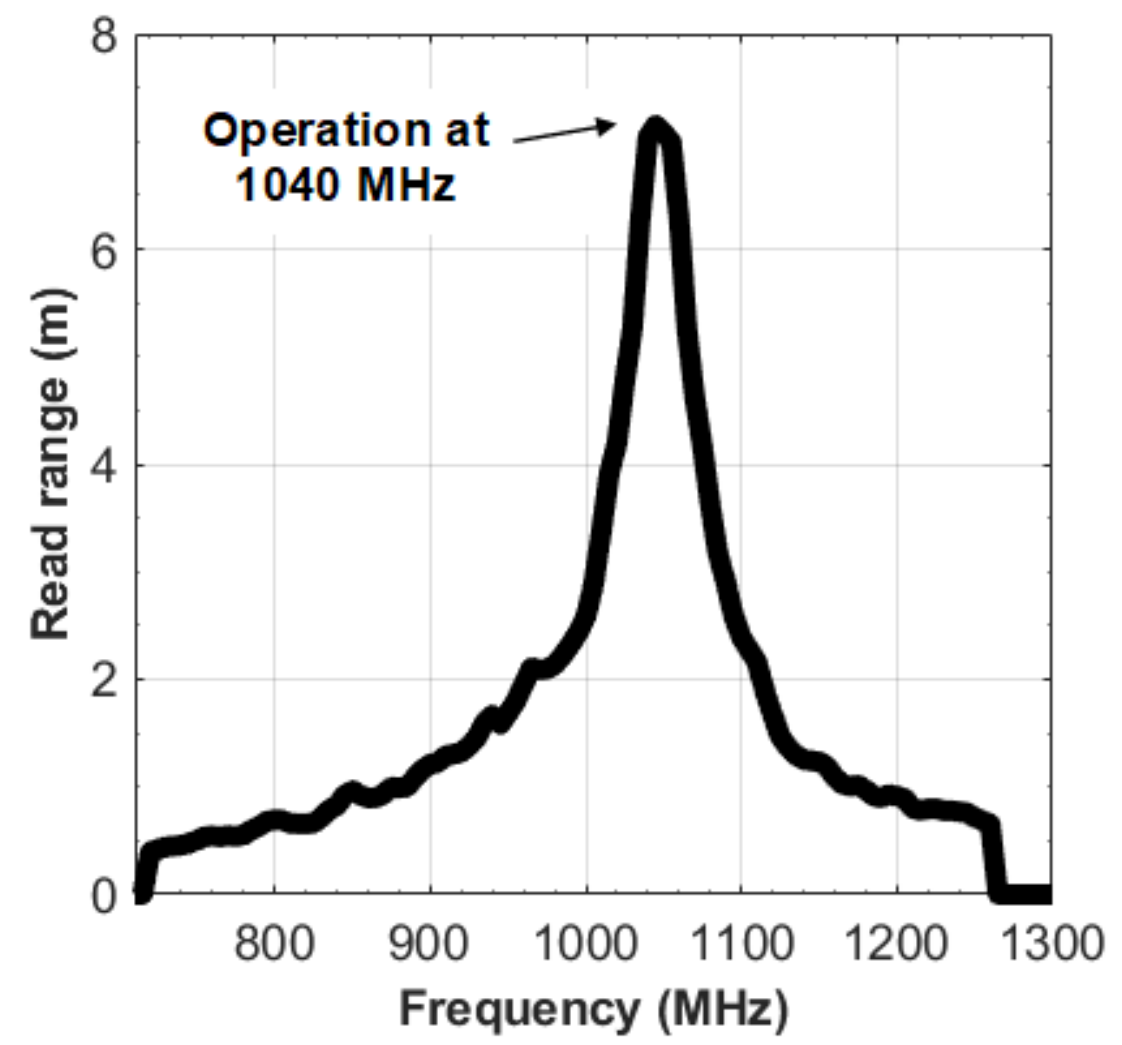

Fig. 13. Measured read range of the helical dipole antenna.

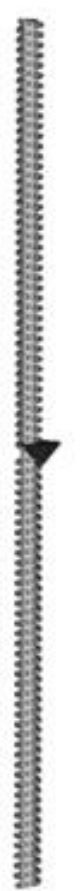

(a)

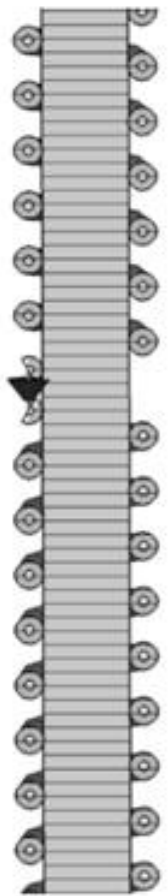

(b)

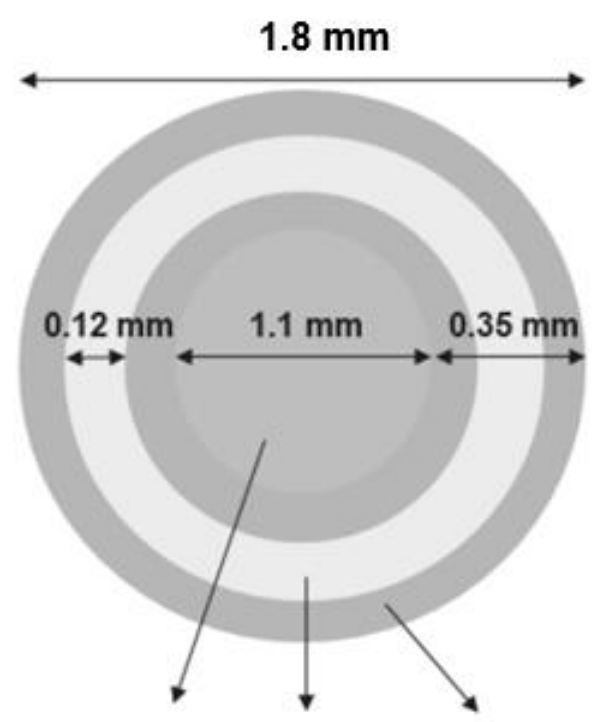

Dielectric core

Antenna

Fig. 14. Geometrical parameters of the simulated manufactured helical dipole antenna: (a) full view of the structure in CST Microwave Studio $®$; (b) sectional view of the structure; (c) front view of the structure. 


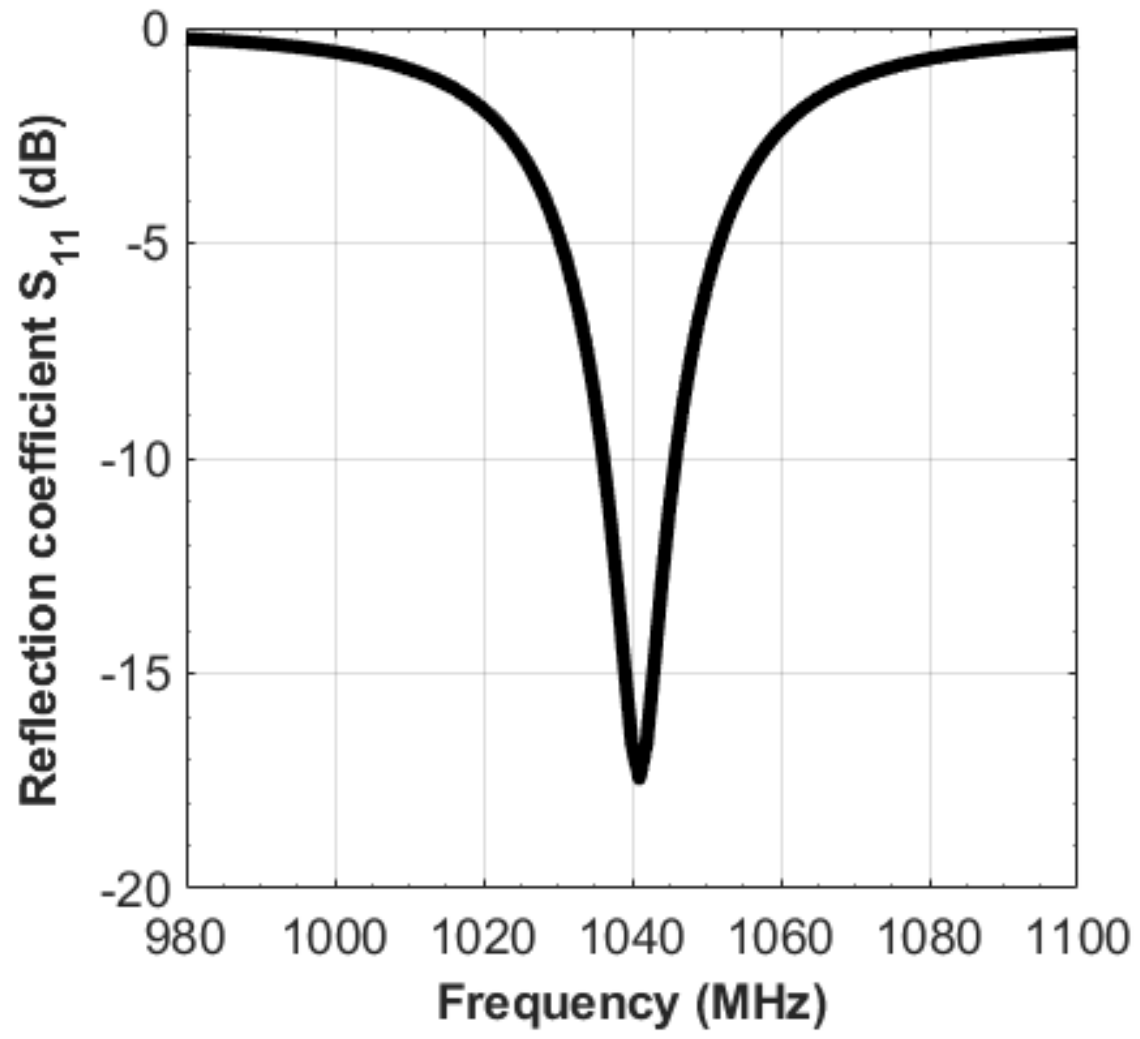

Fig. 15. Simulated reflection coefficient $S_{11}$ of the manufactured helical dipole antenna. 